\title{
Germanica
}

\section{Christian Mariotte, Écrire le stigmate - une nouvelle littérature judéo-allemande}

\section{Martine Benoit}

\section{Q OpenEdition}

\section{$\checkmark$ Journals}

Édition électronique

URL : http://journals.openedition.org/germanica/7258

DOI : 10.4000/germanica.7258

ISSN : 2107-0784

Éditeur

Université de Lille

\section{Édition imprimée}

Date de publication : 26 juin 2019

Pagination : 239-240

ISBN : 978-2-913857-42-1

ISSN : 0984-2632

\section{Référence électronique}

Martine Benoit, "Christian Mariotte, Écrire le stigmate - une nouvelle littérature judéo-allemande », Germanica [En ligne], 64 | 2e trimestre 2019, mis en ligne le 01 janvier 2021, consulté le 02 mars 2021. URL : http://journals.openedition.org/germanica/7258; DOI : https://doi.org/10.4000/germanica.7258 


\section{Comptes rendus de lecture}

Sandie Attia, Signes et traces dans l'œuvre poétique de Günter Eich, Bern et al., Peter Lang (coll. Contacts), 2017, 548 p.

Suivant le tracé d'un Moi protéiforme au statut ambigu, tour à tour producteur ou produit de la parole poétique, Sandie Attia propose dans son étude - dont l'un des multiples mérites est de mettre en lumière la « constellation complexe que forment auteur, textes et lecteur » et ses incidences sur la création - une approche novatrice de l'œuvre poétique et poétologique de Günter Eich à partir des catégories que sont les traces et les signes dont elle fournit dans l'introduction des définitions précises. Jalonnant son propos de poèmes de Eich qui sont autant de preuves à l'appui de ce qu'elle avance, l'auteure entraîne à sa suite le lecteur captivé dans une enquête minutieuse, une collecte d'indices et de traces (intertextuelles, autobiographiques, historiques) dans les écrits de celui qui, paradoxalement, affirmera sa ferme volonté de «ne laisser aucune trace ». La démonstration, étayée par plusieurs études de cas, est d'autant plus riche et convaincante que Sandie Attia puise à des sources inédites (manuscrits, lettres, notes, avant-textes qui font l'objet d'une subtile analyse comparée avec les textes publiés), allant même jusqu'à développer une réflexion originale autour de l'influence des supports sur l'écriture poétique.

La première partie, dans laquelle l'auteure, adoptant un point de vue interne aux textes, procède à une analyse chronologique scrupuleuse des mutations et des migrations des trois motifs que sont les traces dans la neige, les oiseaux-signes et la traduction, apporte un démenti au jugement émis par Hilde Domin qui, dans une lettre datée de 1959, confiait à son mari sa " grande frustration » à la lecture des poèmes de Eich auquel elle reprochait de ressasser un même thème sans jamais 
rien apporter de nouveau. Sandie Attia diagnostique au contraire une évolution, la lente transformation des images poétiques en concepts poétologiques, et montre comment, de glissements en retournements, dans un recours aux effets de cryptage et par un recentrement croissant sur le signifiant au détriment du signifié, s'opère le passage d'une " herméneutique du monde » à une " herméneutique du signe ", de " la lecture des traces au tracé de l'écriture », d'une " poétique de la révélation » fondée sur le décodage des signes à une " poétique de la méditation » entre les signes délestés de toute signification et qui paradoxalement ne font plus même signe vers quelque chose.

$\mathrm{Au}$ croisement de la production poétique et de la réception, la seconde partie ébranle, voire écarte définitivement un certain nombre d'interprétations infléchies, voire biaisées, par « l'intention de lecture d'une époque ", ce dont témoignent les avatars d'« Inventur », poème longtemps considéré comme archétype du Kahlschlag. Si la mise en question de cette lecture n'est pas neuve, l'auteure apporte toutefois un éclairage nouveau en proposant d'aborder le poème non pas comme le paradigme d'une poésie de la captivité, mais comme une « reconstruction a posteriori » de ce que furent les conditions d'écriture de la captivité. Les comparaisons des avant-textes des poèmes rédigés dans le camp de prisonniers avec leur version définitive parue en 1948 dans le recueil Abgelegene Gehöfte permettent d'ailleurs de mettre pleinement en lumière cette notion d'une reconstruction différée d'une réalité passée et revisitée. S'il y a bien un décalage entre les déclarations d'intentions du poète dans ses écrits théoriques de l'immédiat après-guerre - relativement conformes aux attentes des lecteurs ou aux impératifs d'écriture - et la mise en œuvre a posteriori de ces intentions, et si l'oscillation entre continuité et rupture apparaît comme une constante, Günter Eich n'en inscrit pas moins dans ses textes, de façon plus ou moins cryptée, la trace des événements historiques, ainsi que le démontre l'analyse subtile des modifications apportées aux textes des années 1930 repris dans Abgelegene Gehöfte. C'est donc émettre un jugement hâtif que d'affirmer, comme cela fut longtemps le cas, que la poésie de Eich trahirait une indifférence à l'histoire, voire un manque de discernement.

$\mathrm{La}$ " relation dialectique entre tradition et subversion » qui marque la production d'après-guerre transparait également dans les rapports complexes qu'entretenait Eich à Wilhelm Lehmann, poète prônant un escapisme ignorant du désastre, représentatif d'une certaine Allemagne refusant toute confrontation à son passé récent. Si le chapitre consacré à cette relation difficile illustre la façon dont la réception infléchit la production poétique et les positions poétologiques de Eich, il est également une source précieuse de renseignements pour qui serait tenté de suivre les traces de Lehmann, ces sillons " pré-tracés » que le maître voulait imposer et qui aux yeux de son disciple rebelle étaient devenus inopérants. 
La troisième partie débute par l'intention ultime de Eich : "Surtout ne pas laisser de traces ", dont l'auteure rectifie le sens. Il s'agit bien d'un projet poétique et poétologique, et non d'une stratégie d'évitement visant à occulter son propre passé, ce que Eich n'a d'ailleurs jamais tenté de faire (voir à ce propos la longue élaboration de «Wildwechsel », poème en hommage à Nelly Sachs). Suivant le mode d'interprétation génétique, l'auteure procède à une analyse des brouillons successifs de plusieurs poèmes, ces avant-textes qui mettent l'accent sur une « infinité de possibles » qui s'offrent à l'écrivain, avant la fixation dans une version imprimée. Le lecteur devient alors témoin du processus même d'écriture et de la façon dont le poète de la maturité se joue de lui, l'entraînant sur de fausses pistes ou des itinéraires incertains, dans une langue mouvante où « la prolifération des signes » brouille peu à peu « l'accès aux traces ». Le refus de toute traçabilité ne serait-il pas d'ailleurs l'expression d'une suprême liberté du poète, échappant ainsi à toute emprise et à toute instrumentalisation ? Rendre la parole inutile, c'està-dire « inutilisable », y compris par le lecteur, n'est-ce pas là l'aboutissement des efforts déployés avec constance par Günter Eich en vue de l'élaboration, « dans les interstices de la langue asservie », d'un contrelangage irrécupérable privilégiant la langue sur l'énoncé de contenus ?

La thèse de Sandie Attia s'impose d'ores et déjà comme un ouvrage incontournable pour qui veut explorer les écrits de Günter Eich que cet essai magistral fait apparaître comme l'un des grands poètes de langue allemande du siècle dernier, ainsi qu'en témoigne la lettre élogieuse que lui envoya Adorno, alors même que la Frankfurter Allgemeine Zeitung refusait la publication de son discours de Darmstadt, jugé trop dérangeant. Et s'il faut se méfier des traces laissées par le poète, on ne peut à l'inverse que se fier aux pistes dégagées par l'auteure qui convie le lecteur à cette fabuleuse aventure qu'est la découverte d'une œuvre en gestation, du cheminement d'une écriture en devenir dont nous connaîtrons peut-être le dernier mot fixé sur la page, sans avoir pour autant la certitude d'en posséder le sens.

Andrée Lerousseau

Sandra Richter, Eine Weltgeschichte der deutschsprachigen Literatur, München, Bertelsmann, 2017, 728 p.

Sandra Richter, professeure à l'Université de Stuttgart et directrice des Archives littéraires de Marbach depuis 2019, s'intéresse dans cette Histoire mondiale de la littérature germanophone à la circulation et à la réception de celle-ci en dehors des frontières respectives de l'espace culturel germanique depuis le Moyen-âge jusqu'à nos jours. Alors que les histoires littéraires s'inscrivent d'habitude dans une logique natio- 
nale, l'objectif de l'ouvrage est d'apporter un regard globalisé sur la littérature d'une aire linguistique, de voir comment la littérature de langue allemande est perçue ailleurs dans le monde et quelles sont les œuvres canoniques et les traditions littéraires auxquelles on s'intéresse le plus selon les époques. Pour cela, entrent en ligne de compte les différents types de médiateurs et de passeurs, en premier lieu les traducteurs, mais également la critique littéraire et tout autre acteur dans les réseaux de la " médiation culturelle » comme les imprimeurs, les éditeurs ou les gens de théâtre. Ainsi, cette histoire littéraire d'un nouveau genre s'intéresse à la « biographie des livres », au processus de leur migration, la « bibliomigrancy ", à travers le monde (p. 22), en se basant sur des bibliographies des traductions existantes et des statistiques recueillies à cet effet.

Dès le début, l'auteure nous annonce un ouvrage se présentant comme un « patchwork d'exemples de cas » («Flickwerk aus Fallbeispielen») qui ne se veut ni exhaustif - ce qui est impossible vu la période choisie - ni forcément objectif, dans la mesure où il réunit des études de cas « particulièrement passionnantes, importantes ou inconnues » (p. 26) aux yeux de l'auteure. Cette approche éclectique et le manque de critères clairs pour les œuvres retenues se remarquent dès la première période plus largement analysée, allant de 1450 à 1700 . Les premiers exemples retenus font tout à fait sens dans l'optique initialement esquissée : la réception de la Nef des fous de Sebastian Brant, de sa première traduction en latin à l'adaptation par la romancière américaine Katherine Anne Porter en 1962 et la sortie, peu après, du film éponyme, la première traduction anglaise du Eulenspiegel en 1519 ou encore l'adaptation d'œuvres romanesques comme le Faust ou Fortunatus pour des troupes de théâtre anglaises par Christopher Marlowe et Thomas Dekker. Ensuite, on passe à un exemple moins canonique de l'histoire littéraire allemande. Les récits de voyage sur le Brésil que Hans Staden a publiés en 1557 permettant au lecteur germanophone de s'informer sur les coutumes de ces terres lointaines, sont adaptés en livre pour enfants au Brésil du début du $\mathrm{XX}^{\mathrm{e}}$ siècle afin que ceux-ci se familiarisent avec leur propre histoire. On a ici une circulation des influences mutuelles différente d'une simple réception d'un livre de langue allemande en dehors de ses frontières. Il en est de même avec la littérature germano-américaine initiée par les colons allemands à la fin du XVII ${ }^{\mathrm{e}}$ siècle : au lieu de retracer la réception d'une littérature de langue allemande, le chapitre décrit la naissance d'un espace culturel hybride avec des influences variables. Ces exemples sont en effet intéressants, mais faute de feuille de route suffisamment claire, le lecteur se perd facilement dès le début et c'est une tendance qui se confirme dans les chapitres suivants.

Il est évidemment impossible de retracer ici l'ensemble des pièces de ce grand patchwork foisonnant, quelques indications doivent donc suffire. Le XVIII ${ }^{\mathrm{e}}$ siècle est, entre autres, représenté par le fulgurant succès 
de la Mort d'Abel (Tod Abels, 1758) du Suisse Salomon Gessner, auteur d'idylles bucoliques aujourd'hui oublié, une épopée biblique traduite en quatorze langues et qui a eu bien plus de succès que Nathan der Weise de Lessing auquel un autre sous-chapitre est consacré. S'ensuit la période de 1770-1830 qui focalise bien sûr Weimar, de la réception déjà bien connue de Werther à celle de Faust que l'on retrouve aujourd'hui dans des mangas japonais. Est également évoquée la naissance du concept de Weltliteratur forgé par Goethe, compris aujourd'hui comme un canon littéraire globalement reconnu et avec lequel le présent ouvrage prend ses distances. Le XIX ${ }^{\mathrm{e}}$ siècle est résumé de façon étonnante, sur le ton parfois désinvolte de l'ouvrage, comme le siècle du « Drucken, Reisen, Revoluzzen ». Un premier sous-chapitre est consacré au développement de la « Auslandsgermanistik » notamment anglophone, terme quelque peu anachronique pour la période traitée, s'ensuivent des pages sur la réception de l'idéalisme allemand et du romantisme par le cercle des transcendentalistes de Boston autour de Ralph Waldo Emerson. Heine est longuement évoqué comme auteur le plus connu au niveau international après Goethe, quelques pages sont également consacrées à la réception de Karl May ou encore aux sociétés savantes en l'honneur de tel ou tel écrivain germanophone, témoignant de leur influence à l'étranger. Le tournant du siècle est ensuite décrit par la littérature d'inspiration coloniale germanophone, pourtant sans traces de réception à l'étranger, puis par le succès des Buddenbrooks de Thomas Mann. Le début du $\mathrm{XX}^{\mathrm{e}}$ siècle est approché par le rayonnement international de la revue avant-gardiste Der Sturm, par la réception de Rilke qui va jusqu'à devenir un « code » représentant quelque chose de vaguement esthétique, mystique et hermétique dans la culture populaire du XXIe siècle - fallait-il aller jusqu'à inclure une photo montrant un tatoo sur le bras de Lady Gaga reprenant quelques vers des Lettres à un jeune poète? Ce souci d'exhaustivité - que l'on retrouve quelques pages plus loin avec une liste d'auteurs dont l'œuvre se réfère plus ou moins à Kafka - est louable dans la mesure où il souhaite rendre compte du rayonnement de la littérature germanophone jusque dans les confins les plus éloignés de différentes subcultures, mais l'ouvrage court ici le risque de devenir un cabinet de curiosités. L'alignement d'informations sans interprétation plus approfondie laisse par ailleurs un sentiment de frustration.

Avec la période du national-socialisme, l'ouvrage consacre un chapitre à l'exil. En amont, la politique culturelle du Troisième Reich est rapidement mentionnée, l'autodafé du 10 mai 1933 est présenté comme preuve de l'hostilité du national-socialisme à la littérature et à la culture, les étudiants nationaux-socialistes jetant dans les flammes « la littérature des avant-gardes et la modernité littéraire » (p. 306). Mais on aurait aimé lire d'emblée qui étaient les auteurs exclus - à commencer par les juifs -, une écriture moins « moderne» ne les aurait 
pas épargnés. Le chapitre s'intéresse plus particulièrement aux maisons d'édition et aux revues de l'exil, puis à la réception récente de l'œuvre de Stefan Zweig dans un roman graphique français de 2012. On lit ensuite des chapitres sur Rose Ausländer, Paul Celan et Nelly Sachs, sans savoir exactement pourquoi l'accent est mis sur tel ou tel auteur. Vicki Baum est approchée par son succès à Hollywood, Thomas Mann comme l'un des instigateurs de la déclaration « The City of Man. A Declaration on World Democracy » de 1941, Elias Canetti est évoqué à travers ses mémoires sur l'exil londonien publiées de façon posthume en 2003, Party im Blitz, et se retrouve (par association thématique ?) à côté de W.G. Sebald et ses conférences sur la guerre aérienne. Arthur Koestler a également droit au chapitre tout comme Klara Blum, auteure née à Czernowitz et installée en Chine depuis l'après-guerre, dont quelques livres sur la Chine ont été publiés en RDA et qui est aujourd'hui quasiment oubliée. Une trouvaille sans doute intéressante, mais le patchwork se transforme peu à peu en pot-pourri, et l'idée initiale d'écrire une histoire de la « bibliomigrancy » s'éloigne de plus en plus pour devenir une histoire des interpénétrations mutuelles de différentes cultures.

De ce point de vue, les deux derniers chapitres sur la période de 1945 à 1989 et sur l'après-1989 ne sont pas plus convaincants. Pour l'après-guerre, le chapitre s'intéresse aussi bien à la maison d'édition Aufbau et sa volonté de rayonner sur le plan international qu'à Suhrkamp, côté Ouest. Au niveau de la réception de certains auteurs, on trouve Heinrich Böll, Günter Grass et Thomas Bernhard à côté de la seconde réception de Hermann Hesse par les contre-cultures des années 1960 aux États-Unis, ou encore à côté du livre Tumult (2014) de Hans Magnus Enzensberger qui se veut un retour sur la mise en réseaux des différentes avant-gardes de l'Est et de l'Ouest pendant la guerre froide. Là encore, point d'histoire de la réception. Celle-ci est définitivement abandonnée dans le dernier chapitre, car pour la période de l'après-1989, Richter retient - après quelques mots sur la réception anglo-saxonne de la poésie de Durs Grünbein - exclusivement des auteurs de la « littérature interculturelle » : Emine Sevgi Özdamar et Feridun Zaimoglu, Ilija Trojanow et son roman historique Le collectionneur de mondes, Yoko Tawada, Herta Müller et Terézia Mora. Elle clôt ce chapitre avec le roman Boussole du Français Mathias Énard, prix Goncourt 2015, qui est effectivement un exemple de la réception critique de la littérature allemande dans d'autres cultures dans la mesure où il interroge les positions de Goethe dans son Divan occidentaloriental. Mais dans l'ensemble, on se demande pourquoi ce chapitre est exclusivement réservé à la littérature interculturelle. On aurait pu imaginer, par exemple, une réflexion sur l'énorme succès de W.G. Sebald dans le monde anglo-saxon, ou encore sur le succès commercial des Arpenteurs du Monde (2005) de Daniel Kehlmann, deuxième livre 
le plus vendu dans le monde en 2006 et un des plus grands succès d'un livre allemand à l'étranger.

Cette Histoire mondiale de la littérature germanophone se termine avec vingt-cinq thèses censées tirer les enseignements de ce qu'on vient de lire, mais celles-ci ne convainquent pas par leur nouveauté : le lecteur savait probablement à l'avance que " Traduire, c'est s'approprier " (thèse 11) et pouvait se douter qu'un prix Nobel allait augmenter la visibilité d'un auteur à l'international (thèse 14). Autant les premières pages laissaient entrevoir un projet passionnant, autant, le parcours de lecture terminé, l'ensemble paraît très flou et trop subjectif pour aspirer au titre d'une Histoire mondiale de.... Alors que l'auteure avait comme projet initial d'écrire autre chose qu'une histoire littéraire nationale, un des écueils se trouve peut-être dans la décision de suivre de près la périodisation de celle-ci et de vouloir traiter toutes les périodes. L'histoire de la réception mondiale n'aurait-elle pas pu faire surgir d'autres temporalités et d'autres moments forts que ceux déjà bien connus ? Traiter moins d'auteurs et d'événements aurait certainement permis d'aller plus loin dans les analyses, d'introduire des réflexions sur les transferts culturels, concept bien connu mais complètement absent ici. Par ailleurs, pour un ouvrage qui, par son titre, se veut un ouvrage de référence, un travail éditorial plus scrupuleux aurait pu éviter des erreurs factuelles et des approximations fâcheuses. Dans l'ensemble, il s'agit certes d'un livre qui fait découvrir certains aspects moins connus de l'histoire littéraire germanophone et de sa réception dans le monde, mais le patchwork ne s'avère pas aussi stimulant qu'espéré.

\section{Carola Hähnel-Mesnard}

Alexandre Dupeyrix et Gérard Raulet (éd.), Allemagne 1917-1923 - Le difficile passage de l'Empire à la république, FMSH-Éditions, Paris, 2018, 164 p.

Inscrit dans le jubilé du centenaire du premier conflit mondial, cet ouvrage collectif dirigé par Gérard Raulet et Alexandre Dupeyrix propose d'éclairer des aspects encore mal connus et qui, pour certains, réinterrogent des idées reçues, sur cette période décisive du passage de l'Empire à la première république allemande - une république qui doit assumer les conséquences de la guerre, notamment la perte de $13 \%$ du territoire, du dixième de sa population, de la totalité de ses colonies et la réduction de son armée. On est, comme le soulignent les directeurs de l'édition, dans une " configuration historique inédite » avec « à l'extérieur l'effondrement des grands empires » et à l'intérieur « de nombreuses résistances » à l'instauration de la république (1). Les contributeurs (on pourra regretter ici l'absence de notices de présenta- 
tion) proposent tour à tour des exposés larges (sur le chemin menant au Traité de Versailles, sur la difficile mise en place du parlementarisme, sur les mesures de protection de la République, sur le concept de dictature) et des développements sur de grandes figures de Weimar : Ernst Troeltsch, Ernst Toller et Oskar Maria Graf, Max Weber - deux contributions présentant quant à elle des personnalités restées moins connues : Sigmund Rubinstein et son projet romantique d'une république des conseils; le rôle des féministes radicales dans le processus de transition démocratique.

Vincent Laniol (Université Paris 1) retrace « la longue route vers Versailles ", il souligne notamment que ce sont bien Ludendorff et Hindenburg qui demandent l'armistice (« [...] seule la direction de l'armée fut responsable de la demande de paix » (4) : l'affirmation du coup de poignard dans le dos lancée par Hindenburg est bien une légende). Vincent Laniol montre également que les Allemands étaient prêts à des concessions financières (le chiffre de 100 milliards de marksor est avancé) dans l'espoir d'une révision des clauses territoriales, et rappelle la présence des gueules cassées imposée par Clémenceau à la signature du traité de paix dans la Galerie des Glaces le 28 juin 1919.

Gérard Raulet (Université Paris-Sorbonne) se penche sur le républicanisme de raison (Vernunftrepublikaner) par l'exemple de Ernst Troeltsch et de ses « Spectator-Briefe », chroniques de culture politique parues entre février 1919 et juillet 1920 puis jusqu'en octobre 1922 sous le titre de Berliner-Briefe (Troeltsch avait alors mis un terme à l'anonymisation et signait de son nom) dans le Kunstwart. Membre du DDP, élu à la Preussische Landesversammlung en 1919, sous-secrétaire d'État auprès du Kultusministerium de Prusse, Troeltsch fait partie de ces républicains qui défendront « avec acharnement la position qui a été celle des pères de la constitution de Weimar, le 'système de Weimar', une coalition de compromis allant des sociaux-démocrates aux libéraux de la DDP » (30). Pour Troeltsch, la république démocratique est bien « irréversible et nécessaire » (36) et doit être une sorte de refondation de l'Empire (« Il faut reconstruire à nouveaux frais l'administration et la constitution tant de l'Empire que de ses éléments » (36), écrit-il en août 1919) dont elle doit absolument assurer l'unité : aussi la démocratie ne pourra-t-elle être qu'« unitariste et centralisatrice » (38).

S'intéressant à la parlementarisation du Reich, Horst Möller (Université Ludwig Maximilian Munich) rappelle l'importance grandissante des partis dans la vie politique sous l'Empire, qui explique l'émergence en 1919 de la coalition de Weimar constituée du SPD, du Zentrum, du DDP et du DVP de Gustav Stresemann. Horst Möller rappelle en outre qu'" avec l'introduction du droit de vote pour les femmes au sein d'un système proportionnel, le mode de scrutin du Reichstag et des parlements des Länder devint le plus démocratique et le plus progressiste d'Europe » (54-55). L'auteur insiste 
sur le rôle des hommes et des femmes dans l'engagement démocratique et sur la fragilisation du processus de parlementarisation avec la disparition prématurée de Friedrich Ebert et de Gustav Stresemann et les assassinats de Hugo Haase, Matthias Erzberger et Walther Rathenau, notant que les assassinats de Rosa Luxemburg, Karl Liebknecht et Kurt Eisner ont, eux aussi, joué un rôle funeste car " même si ces derniers ne soutenaient pas le parlementarisme, ils auraient peut-être pu empêcher que le KPD ne suive par la suite la ligne du Komintern, entièrement décidée par Moscou » (59). Christian E. Roques (Université de Reims Champagne-Ardenne) revient sur la publication fin 1920 de l'ouvrage de Sigmund Rubinstein, Romantischer Sozialismus. Reprenant l'idée de l'institution des conseils ouvriers dans une logique cependant clairement antibolchévique, Rubinstein renvoie dos à dos marxisme et militarisme et affirme une conception allemande, expression d'un socialisme créateur, revenant à la démocratie directe, qu'il estime être la forme originelle de la démocratie. La démocratie se doit d'être autogérée, l'économie d'être coopérative.

Cécile Poncet (Université Paris-Sorbonne) nous livre un très beau portrait artistique et politique croisé de Ernst Toller et Oscar Maria Graf en revenant sur ces républiques (bavaroises) des écrivains en avril 1919 dont la répression par les corps francs fit plus de 1000 assassinés, 2000 arrestations et plus de 5000 procédures pénales (on soulignera ici l'excellente idée d'ouvrir ce livre collectif à de jeunes mastérisants). Toller, qui, aux côtés d'Erich Mühsam et Gustav Landauer, met son engagement au service de Kurt Eisner, regretta ultérieurement son engagement : «Cette république des conseils était une erreur, une erreur, on doit la reconnaître et la supprimer » (93). On rappellera en outre que Brecht écrivit un de ses plus beaux poèmes, "Die Bücherverbrennung ", en hommage à Oscar Maria Graf qui, horrifié d'apprendre que son œuvre avait été épargnée lors des autodafés de mai 1933, avait publié un article en forme d'injonction aux nazis, « Brûlez-moi », dans le journal viennois Die Arbeiterzeitung.

Nathalie Le Bouëdec (Université de Bourgogne) montre que, contrairement à l'idée reçue d'une république sans défense (109), la république de Weimar a tenté de combattre légalement la violence politique illégale dont elle était l'objet, l'assassinat de Walter Rathenau ayant joué ici le rôle de déclencheur (et N. Le Bouëdec évoque la " vague d'émotion sans précédent dans l'opinion publique et la classe politique » (102) à la suite de cet assassinat). Rappelant le travail de documentation remarquable d'Emil Julius Gumbel, l'article se penche sur la Gesetz zum Schutz der Republik du 21 juillet 1922, « tentatives pour créer une conscience civique républicaine, ancrer les valeurs de la république chez les citoyens et tarir la violence politique à sa source » (107).

Anne-Laure Briatte (Université Paris-Sorbonne) nous invite à une analyse de la contribution des intellectuelles féministes radicales à la 
transition politique entre 1890 et 1920, avec des femmes comme Minna Cauer, Anita Augspurg (première Allemande à devenir Docteure en droit), Lida Gustava Heymann, Helene Stöcker. Revendiquant l'égalité juridique entre les sexes et le droit de vote des femmes, elles saluent la proclamation du droit de vote direct, égal et secret pour les hommes et les femmes: «Rêve de ma jeunesse, exaucé au soir de ma vie ! Je meurs républicaine » écrit ainsi Minna Cauer dans son Journal le 9 novembre 1918. Les années de Weimar sont des années d'engagement pour une paix durable, le droit des peuples à disposer d'eux-mêmes, et toujours et encore l'égalité entre les sexes. En 1933, « féministes, démocrates et pacifistes » (127), Anita Augspurg, Lida Gustava Heymann, Helene Stöcker fuient l'Allemagne.

Marcus Llanque (Université d'Augsbourg) se penche sur le concept de dictature et son lien avec celui de démocratie, le terme de dictature permettant « de se pencher sur la manière dont les régimes prédémocratiques pouvaient donner naissance à des démocraties » (132). Sont ainsi analysées les notions de "dictature du prolétariat », de " dictature du président » qui « ont pour point commun de mettre au jour les limites de la démocratie » (144). Marcus Llanque souligne ainsi avec soin que, dans une acception positive, la dictature du président avait pour but de protéger la démocratie et la dictature du prolétariat de la faire triompher et avancer : « le vrai problème, celui du règlement démocratique de la démocratie, est resté » (145).

Alexandre Dupeyrix (Université Paris-Sorbonne) nous présente finalement deux conférences célèbres de Max Weber, "Wissenschaft als Beruf » (prononcée le 7 novembre 1917) et «Politik als Beruf » (tenue le 28 janvier 1919) dans lesquelles la formule du « combat des dieux » et les concepts d'" éthique de conviction » et " éthique de responsabilité » sont introduits et qui sont nourries des événements qui ont marqué Weber. Alexandre Dupeyrix rappelle avec pertinence que Weber témoigna au procès intenté à Ernst Toller et que c'est son développement sur la notion d'éthique de conviction qui évita à l'écrivain engagé la peine capitale. Adhérent du DDP entre 1918 et 1920, Weber expérimente les difficultés du philosophe engagé incapable de devenir un véritable homme politique et cherche à définir " le rôle de la science et de l'érudit dans l'actualité politique » (159). Si l'éthique de conviction, justifiant les moyens par la fin, élude « la responsabilité des conséquences », l'éthique de responsabilité prend en compte la réalité sociale et politique : «[...] l'homme politique véritable est précisément celui qui sait articuler [les deux] » (161).

S'il fallait relever les lignes de force de cet ouvrage collectif de très grande qualité, ce serait cette importance de l'engagement, pour le combat démocratique, de personnalités irremplaçables et dont la mort prématurée (Ebert), l'assassinat (Rathenau) ou les échecs (Toller, mais 
aussi Anita Augspurg, Lida Gustava Heymann, Helene Stöcker) ont été fatals à cette première république allemande.

Martine Benoit

Myriam Bienenstock, Cohen und Rosenzweig. Ihre Auseinandersetzung mit dem deutschen Idealismus, Freiburg/ München, Verlag Karl Alber, 2018, 298 p.

L'essai de Myriam Bienenstock, qui propose ici une version revue et augmentée de son étude parue en français en 2009, répond à la nécessité, formulée par Emil Fackenheim dans son « Épitaphe pour le judaïsme allemand », qu'il y aurait à se pencher sur le « Siècle d'Or» de la philosophie allemande si l'on veut restituer pleinement et maintenir vivant l'héritage de Hermann Cohen (1842-1918) et de celui qui fut son élève, Franz Rosenzweig (1886-1929), l'un et l'autre étant trop souvent appréhendés comme étant essentiellement des penseurs juifs dont l'œuvre puiserait en priorité dans la tradition juive. L'essai s'attache ainsi à analyser d'une part la compréhension que ces philosophes ont de l'idéalisme allemand et d'autre part la façon dont ils débattent entre eux sur la base de cette philosophie, apportant ainsi leur contribution à l'histoire des idées en Allemagne.

L'essai - et ce n'est pas la moindre de ses qualités - est construit avec une remarquable rigueur : après le rappel d'un certain nombre de données biographiques, chaque chapitre, placé sous le signe du questionnement, se déploie en trois parties, permettant à l'auteure à la fois d'analyser la façon dont s'élabore la pensée des deux philosophes dans un débat permanent avec l'idéalisme allemand, leur appréhension étant parfois infléchie par la réception de celui-ci chez leurs contemporains (Dilthey ou, pour ce qui concerne Rosenzweig, Meinecke). Cette lecture " orientée » est pour l'auteure l'occasion d'exercer sa propre réflexion critique. Ainsi Myriam Bienenstock se fait-elle à maintes reprises l'avocate de Hegel, lorsqu'elle s'inscrit par exemple en faux contre « l'eschatologisation » de la pensée hégélienne à laquelle procède Rosenzweig sous l'influence d'Ehrenberg, rappelant que la visée ultime de Hegel n'était nullement la Rédemption, mais la liberté politique, et elle n'hésite pas non plus à revisiter l'image de Cohen esquissée par Rosenzweig et reprise par Löwith, tendant à faire également du messianisme de Cohen une eschatologie, le prophétisme et le messianisme étant bien plutôt chez Cohen, soucieux de maintenir à l'horizon un idéal politique socialiste, au fondement d'une " éthique sociale ». L'auteure souligne en outre la lecture quelque peu biaisée que fait Rosenzweig de la philosophie politique de Hegel au prisme de l'enseignement de Meinecke, même si Rosenzweig parvient à se démarquer de son maître. 
Dans sa critique de l'idéalisme transcendantal dirigée aussi contre Hegel, Rosenzweig semble enfin ignorer la critique émise par le philosophe lui-même à l'encontre de cet idéalisme.

Nourries de leur confrontation avec le christianisme d'une part et l'idéalisme allemand d'autre part, la philosophie de Cohen et la " pensée nouvelle » de Rosenzweig s'édifient en particulier à partir de ces pierres d'achoppement que sont pour ces penseurs juifs la tentation de représenter Dieu et l'anthropomorphisme dans l'art, le panthéisme hérité de Spinoza, la notion de médiation ou d'incarnation, la philosophie du moi développée par Fichte et de l'intériorité chère à l'école romantique, la réduction - à l'exception peut-être de Herder - de la langue au logos ou plus exactement à une logique étrangère à la langue, le mythe et la « mythologisation » de l'histoire dans l'historisme dénoncé par Rosenzweig, etc. Le débat autour du panthéisme responsable de nombre de maux et l'approche critique à la fois de Spinoza et de la lecture de Spinoza effectuée par Schleiermacher et par l'école romantique (imposant à Cohen de rétablir la vérité sur le philosophe) ou par Dilthey, héritier de l'idéalisme, ayant décelé chez Hegel un "panthéisme de l'esprit », occupe une place importante du début à la fin de l'essai et témoigne du souci de contextualisation : à la discussion autour du spinozisme et du panthéisme succèdera assez tardivement chez Cohen la critique - radicalisée chez Lévinas - du Traité théologico-politique entreprise dans le contexte de la montée de l'antisémitisme dont le discours trouvait une source inépuisable dans l'antijudaïsme contenu dans le Traité. C'est également dans ce contexte et celui de la Première Guerre mondiale que Cohen, se faisant le défenseur du judaïsme, rappelle le message relatif à l'amour du prochain ou plus précisément au caractère infondé de toute forme de haine contenu dans la Bible et le Talmud, et que Rosenzweig tente d'élaborer avec son cercle d'amis une " pensée nouvelle » en rupture avec l'hégélianisme (sans voir à l'instar de Meinecke dans la philosophie politique de Hegel une philosophie prônant un État fort au sens où l'entendra Bismarck ou une apologie du nationalisme, Rosenzweig n'en soulignait pas moins qu'avec Hegel s'amorçait une évolution dans cette direction).

Ces pierres d'achoppement sont pour Cohen et Rosenzweig l'occasion d'entamer, à partir de leur propre perception de la tradition juive, une réflexion sur l'esthétique (voir le chapitre consacré à l'analyse de l'ironie chez Cohen et le rappel de l'esthétique développée par Rosenzweig dans L'Étoile de la Rédemption), et de proposer un certain nombre de redéfinitions : celle du concept « d'autonomie » chez Cohen, une autonomie non plus comprise comme détermination de soi par le soi (la « Selbstbestimmung » fichtéenne) mais fondée sur une « corrélation » entre Dieu et l'homme, entre loi et devoir, et qui, en dépit de l'extériorité maintenue de la loi, n'est pas sans présenter une certaine 
parenté avec la conception de Kant selon laquelle ce n'est pas le concept de liberté qui fonde l'impératif catégorique, mais l'inverse ; ou celle de l'esprit chez Rosenzweig et d'une science de l'esprit qui s'édifie sur l'analyse de la langue dont la base est le « Tu », sur cette "sphère » dont Rosenzweig déplore l'absence dans l'idéalisme allemand et même chez Schelling, philosophe qu'il admire. Autant de considérations qui déboucheront sur une philosophie de l'interpellation et de la responsabilité qui culminera chez Lévinas.

Le dernier chapitre qui s'applique d'ailleurs à dégager les traces de Cohen et de Rosenzweig dans l'œuvre de Lévinas, est particulièrement stimulant. Outre une analyse passionnante des écrits de ce philosophe, l'auteure y fait la démonstration que la pensée juive contemporaine ne peut s'appréhender sérieusement que dans une prise en compte de l'héritage de ce judaïsme allemand ancré dans une réflexion autour des deux traditions, juive et allemande, et dont Hermann Cohen et Franz Rosenzweig furent les plus éminents représentants. Le débat semble d'ailleurs se poursuivre, ainsi qu'en témoigne la réponse de Derrida au jugement prononcé par Lévinas sur Heidegger. Il y a quelque chose, dans ce rapport entretenu par les penseurs juifs à la tradition philosophique allemande, qui n'est pas sans rappeler la relation que dit avoir Claude Vigée, poète et essayiste, à Hegel lorsqu'il qualifie celui-ci « d'ennemi intime »: une intimité qui n'impliquerait pas nécessairement une parenté, encore moins une symbiose, mais une fréquentation constante permettant de mieux se situer.

Andrée Lerousseau

Christian Mariotte, Écrire le stigmate - une nouvelle littérature judéoallemande, Presses Sorbonne Nouvelle, Paris, 2018, 253 p.

L'ouvrage de Christian Mariotte interroge la notion de littérature judéo-allemande à travers l'étude des œuvres littéraire, autobiographique et critique de trois auteurs, Barbara Honigmann, Maxim Biller et Rafael Seligmann. Abordant cette écriture en sociologue, à l'instar de Clara Lévy à laquelle il fait explicitement référence (pour son livre Écritures de l'identité - Les écrivains juifs après la Shoah, 1998), Christian Mariotte s'appuie sur la notion de « stigmate » développée au milieu des années 1970 par le sociologue américain Erving Goffman. Si on peut parfois un peu regretter cette approche qui semble faire obstacle à des analyses littéraires plus poussées, on ne peut qu'être admiratif devant l'ampleur de la tâche que s'est fixée Christian Mariotte en abordant, à partir du croisement de l'étude des textes des trois auteurs, des sujets aussi divers que la famille, l'institution scolaire, la réunification, l'antisémitisme, le lien avec les institutions juives, le 
rapport à Israël, le philosémitisme littéraire, la mémoire de la Shoah, le reproche d'instrumentalisation de la souffrance, le lien à la langue allemande - et cela du milieu des années 1980 aux années 2010. Christian Mariotte rappelle d'emblée la " double marginalité » (23) des Juifs restés en Allemagne : marginaux vis-à-vis de la société allemande, marginaux vis-à-vis de la communauté juive mondiale. Le travail de croisement des œuvres, leur confrontation permet de montrer les lignes de partage et de divergence des trois auteurs dont le regroupement est d'une grande cohérence : écrivains de la deuxième génération dont les parents ont émigré puis sont revenus en Allemagne, ils assument une identité juive dans des confrontations souvent directes avec leur environnement. Ainsi s'exprime à travers leurs œuvres une oscillation entre une littérature de haine et une littérature de réconciliation, écart qui n'est parfois que question de maturité, notamment chez Maxim Biller qui, après avoir écrit pendant des années ses « chroniques de la haine », en revient pour amorcer une littérature de l'apaisement : il évoque ainsi une « humeur paranoïaque » (189) dont il aurait réussi à se détacher. S’il fallait trouver une formule pour chacun de ces trois auteurs, on pourrait dire de Seligmann qu'il est le plus torturé, de Honigmann qu'elle est la plus apaisée, de Biller qu'il est le plus expressif. Christian Mariotte remarque en outre que, du point de vue littéraire, c'est certainement Barbara Honigmann qui est l'auteure la plus aboutie des trois.

Je garde quant à moi un faible pour l'humour grinçant de Maxim Biller, pour son sens de la formule, quand il traite les antisémites de «Stürmer du dimanche » (51) ou quand il décèle derrière l'antisionisme un antisémitisme mal assumé : « Et ainsi, nous en venons à parler d'Israël. C'est-à-dire que je parle d'Israël et qu'eux, comme d'habitude, ne parlent que de leur propre problème. Ils disent sionisme et ils veulent dirent NSDAP, ils disent Sharon et ils veulent dire Eichmann » (140 texte paru en 2001).

On lira avec profit cette étude riche et approfondie qui aborde souvent avec finesse des problématiques délicates. Christian Mariotte donne envie de relire les textes de Honigmann, Seligmann et Biller. 